\title{
Pathology turned its back on semiology
}

\author{
Sara Barreto ${ }^{1}$, Ana Domingos ${ }^{2}$, David Navarro ${ }^{3}$, Mário Góis ${ }^{3,4}$, Helena Sousa ${ }^{3,4}$, Fernando Nolasco ${ }^{3}$ \\ ${ }^{1}$ Nephrology Department, Hospital Garcia de Orta, Almada \\ 2 Nephrology Department, Centro Hospitalar e Universitário do Algarve, Faro \\ 3 Nephrology Department, Hospital de Curry Cabral, Centro Hospitalar e Universitário de Lisboa Central \\ ${ }^{4}$ Laboratory of Renal Morphology, Hospital de Curry Cabral, Centro Hospitalar e Universitário de Lisboa Central
}

\section{CLINICAL PRESENTATION}

We present a case of a 32-year-old caucasian female with systemic lupus erythematosus (SLE) diagnosed 7 years ago. At that time, patient had severe arthritis (hands, ankles and knees), cutaneous involvement (petechiae) and was treated with deflazacort and hydroxychloroquine. There were no signs of kidney damage (normal serum creatinine, inactive urinary sediment and no proteinuria). The following years were uneventful, until one year ago, when patient presented with worsening of arthritis. Bloodwork showed normochromic normocytic anemia ( $\mathrm{Hb} 10.9 \mathrm{~g} / \mathrm{dL})$, leukopenia $(4.33 \mathrm{x}$ $\left.10^{\wedge} 9 / \mathrm{L}\right)$, normal platelet count, normal serum creatinine $(\mathrm{Cr} 0.73$ $\mathrm{mg} / \mathrm{dL}$ ), positive antinuclear antibodies (ANA 1:640), positive double-stranded DNA antibody (anti-dsDNA $1509 \mathrm{UI} / \mathrm{ml}$ - ELISA), positive anti-glomerular basement membrane antibody (anti-GBM $36.4 \mathrm{UQ}$ ), low C3 (0.59 g/L; reference range $0.9-1.8 \mathrm{~g} / \mathrm{L})$ and low C4 levels $(0.08 \mathrm{~g} / \mathrm{L}$; reference range $0.1-0.4 \mathrm{~g} / \mathrm{L})$. Laboratory tests also showed elevated urinary protein to creatinine ratio $1135 \mathrm{mg} / \mathrm{g}$ and presence of dysmorphic erythrocytes in urinary sediment.

A kidney biopsy was performed.

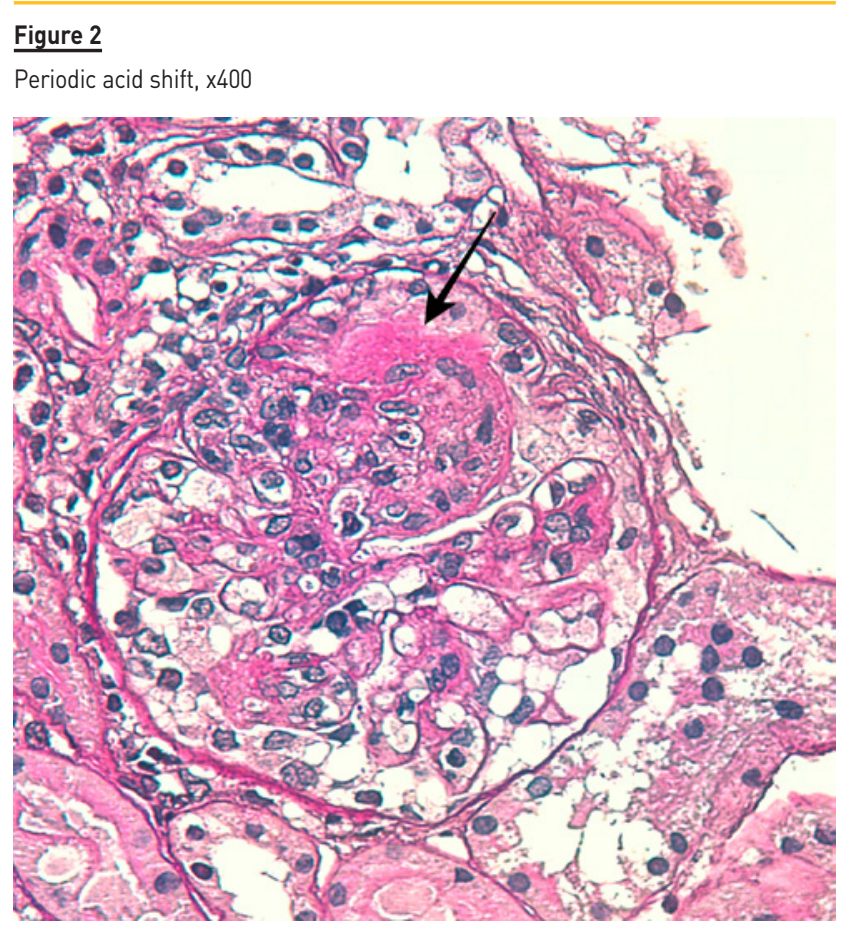

Figure 3

Methenamine-Silver, $x 400$

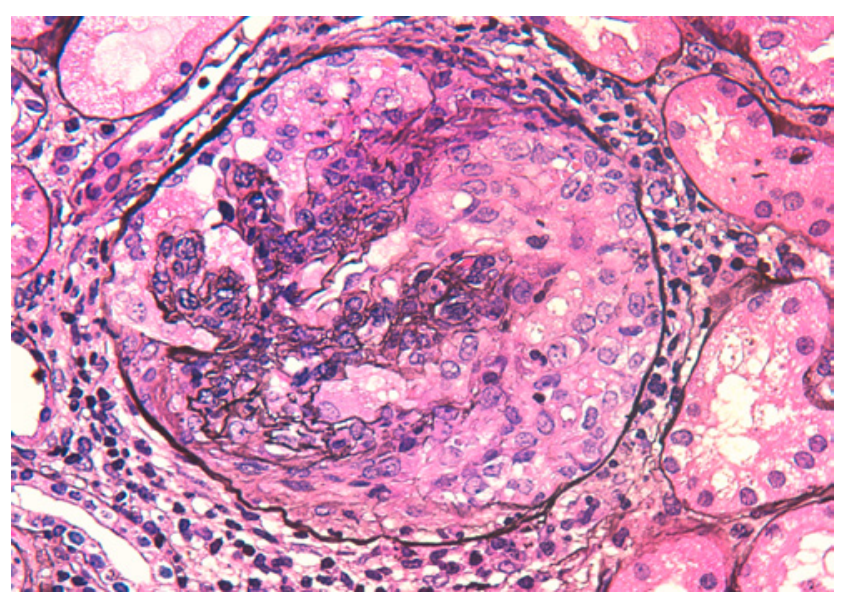




\section{Figure 4}

Methenamine-Silver, $\times 400$

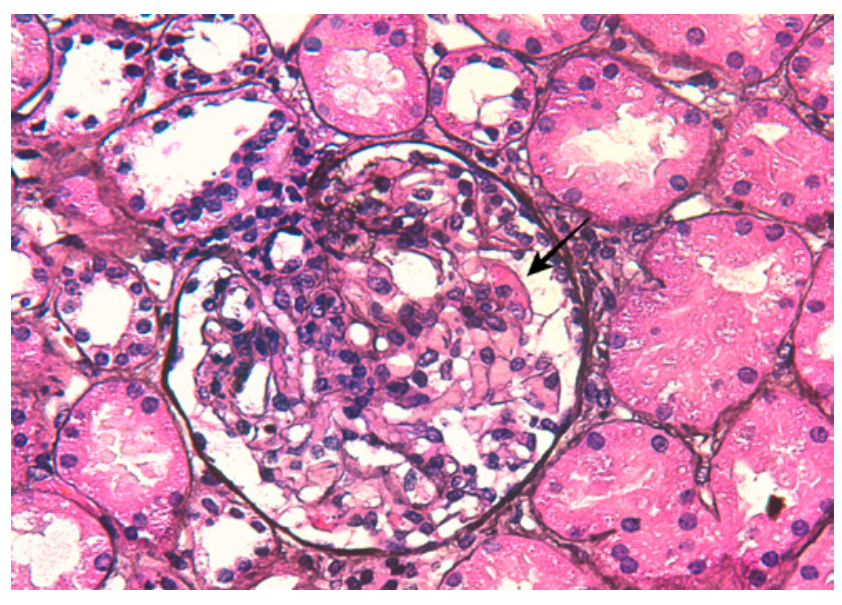

\section{Figure 5}

Periodic acid shift, $x 400$

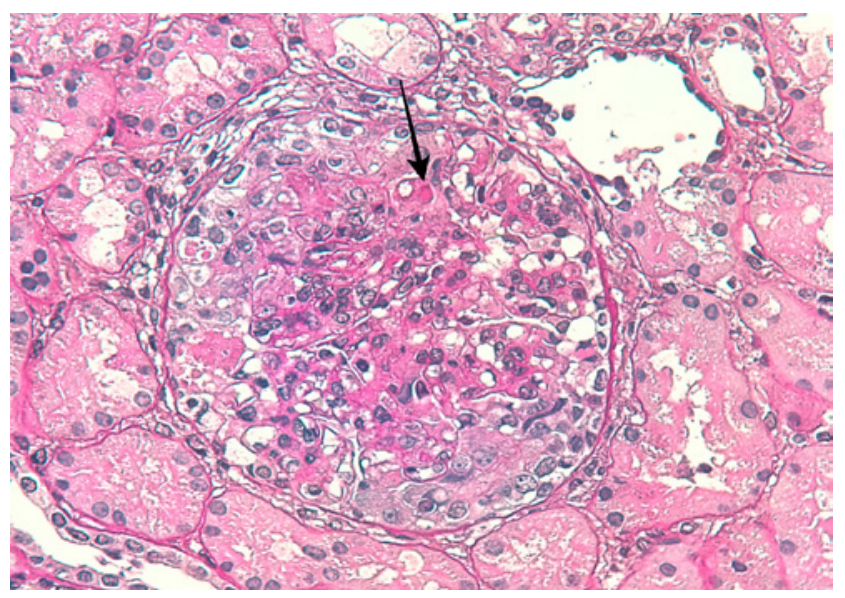

\section{QUESTIONS}

1. According to the clinical, serological and morphological features presented, what is the most likely diagnosis?

2. What is the activity/chronicity index?

3. According to the new classification system, what is the prognosis?

4. Would you perform a kidney biopsy in this patient?

\section{Figure 6}

Immunofluorescence (paraffin embedded tissue)

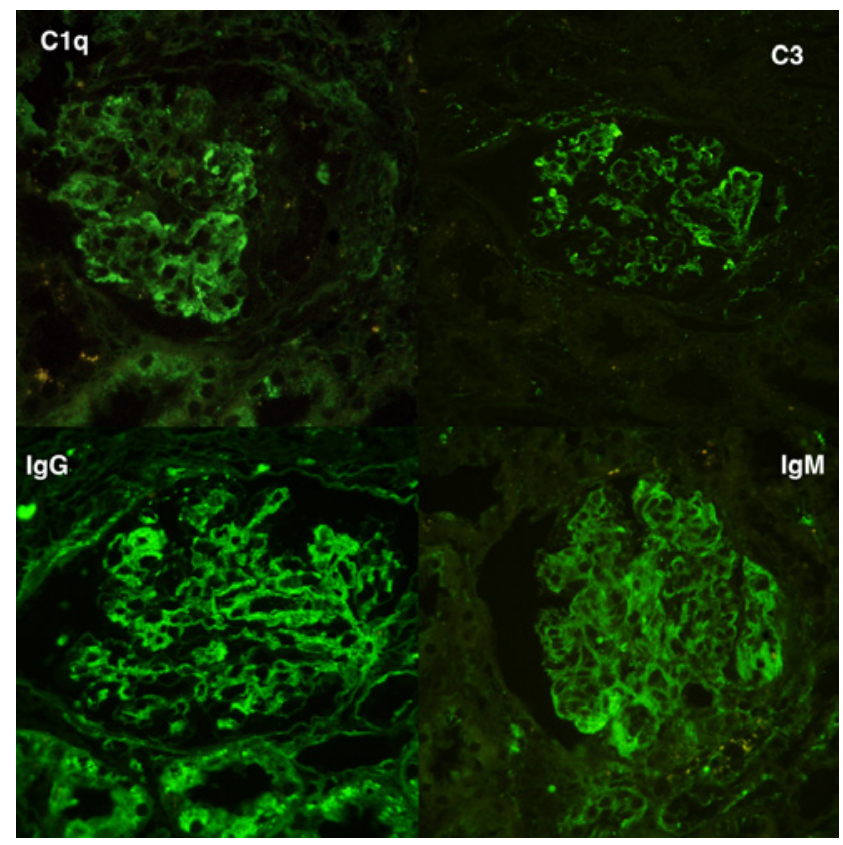

\section{ANSWERS}

1. According to the clinical, serological and morphological features presented, what is the most likely diagnosis?

The fragment obtained from kidney biopsy consisted of renal capsule, cortical with 23 glomeruli, medium sized-arteries and medulla. In light microscopy, podocyte hyperplasia, mesangial and endocapillary hypercellularity with neutrophils (Figure 1, square), as well as mesangial expansion, were evident in virtually all glomeruli sampled. Fibrinoid necrosis (Figure 2, arrow) and cellular crescents were present in 6 glomeruli (Figure 3). Some had hyaline subendothelial deposits (Figure 4, arrow), thrombi (Figure 5, arrow) and cellular debris, compatible with karyorrhexis (Figure 1, arrow). The tubulointerstitial compartment was well preserved and no significant changes were noted in the observed arteries. The immunofluorescence (IMF) was performed in paraffin embedded tissue and documented deposition of IgG (+++), $\operatorname{lgA}(+), \operatorname{lgM}(++)$, C3 (+++), C1q (++), kappa (++) and lambda (++) light chains in the mesangium and parietal aspect of the glomerular capillaries (Figure 6).

The morphological features encountered, together with clinical and serological evidence of SLE, support the diagnosis of Lupus Nephritis (LN). Renal disease is a common manifestation in SLE, affecting up to $60 \%$ of patients during its course. ${ }^{1}$ Moreover, the finding of a full house pattern in IMF along with widespread glomerular hypercellularity in a patient with SLE strongly suggests LN. ${ }^{2}$ In this case, the presence of endocapillary and extracapillary hypercellularity in more than $50 \%$ of the glomeruli (previously defined as diffuse involvement) classifies it as a Class IV LN. 


\section{Table 1}

Proposed modified NIH lupus nephritis activity and chronicity scoring system.

\begin{tabular}{|c|c|c|}
\hline Modified NIH activity index & Definition & Score \\
\hline Endocapillary hypercellularity (\% of affected glomeruli) & \multirow{6}{*}{$<25 \%(1+) 25-50 \%(2+)>50 \%(3+)$} & $0-3$ \\
\hline Neutrophils/karyorrhexis (\% of affected glomeruli) & & $0-3$ \\
\hline Fibrinoid necrosis (\% of affected glomeruli) & & $(0-3) \times 2$ \\
\hline Hyaline deposits - Wire loop lesions / hyaline thrombi (\% of affected glomeruli) & & $0-3$ \\
\hline Cellular/fibrocellular crescents (\% of affected glomeruli) & & $(0-3) \times 2$ \\
\hline Interstitial Inflammation - Leukocytes (\% of affected cortex) & & $0-3$ \\
\hline Total & & $0-24$ \\
\hline Modified NIH chronicity index & Definition & Score \\
\hline Total glomerulosclerosis score - Global and/or segmental (\% of affected glomeruli) & \multirow{4}{*}{$<25 \%(1+) 25-50 \%(2+)>50 \%(3+)$} & $0-3$ \\
\hline Fibrous crescents (\% of affected glomeruli) & & $0-3$ \\
\hline Tubular atrophy (\% of cortical tubules) & & $0-3$ \\
\hline Interstitial fibrosis (\% of affected cortex) & & $0-3$ \\
\hline Total & & $0-12$ \\
\hline
\end{tabular}

\section{What is the activity/chronicity index?}

The modified National Institute of Health (NIH) activity and chronicity scoring system ${ }^{3}$ assigns a maximum of 24 points for total activity and 12 points for chronicity (Table 1).

In this case there is a total score of 15 in the activity index: endocapillary hypercellularity and karyorrhexis in more than $50 \%$ of glomeruli ( $3+3$ points), fibrinoid necrosis and cellular crescents in $25-50 \%$ of glomeruli ( $4+4$ points), hyaline deposits in less than $25 \%$ of glomeruli (1 point) and no interstitial inflammation was observed (0 points). There were no criteria for chronic lesions.

\section{According to the new classification system, what is the prognosis?}

Currently, it is accepted that the clinical outcome of different classes is variable, with heterogeneity within the classes themselves. ${ }^{4}$ Early diagnosis and treatment of LN improves prognosis. ${ }^{5}$ Additionally, several data suggest worse outcomes in black and Hispanic patients compared with non-Hispanic white patients, in proliferative LN and in inappropriately treated classes III and IV. ${ }^{4,5}$

Several recent reviews ${ }^{4,6-8}$ have identified new putative prognostic factors, namely fibrous crescents, fibrinoid necrosis, vascular and tubulointerstitial lesions. The latter is potentially the most significant prognostic indicator. ${ }^{8,9}$ The need for revision of $\mathrm{NIH}$ scores came mainly from limitations regarding reproducibility and prognostic value. $3,6,7,10$ In addition to the above mentioned features, another important modification introduced by this proposed scoring system is related to the elimination of segmental and global subdivisions of class IV, due to unclear definitions, interobserver variability and uncertain clinical significance. ${ }^{3}$ Lack of prediction of treatment response is still a concern. ${ }^{7,10}$ Data regarding chronic lesions in the postinduction biopsy also seem to play a role in defining long-term renal outcomes ${ }^{5}$, although indications to repeat biopsy are still controversial. ${ }^{4}$ Salem Almaani and colleagues ${ }^{10}$ now suggest the inclusion of molecular data, adding independent diagnostic and prognostic information.

In this particular case, despite the presence of fibrinoid necrosis and hyaline deposits - which might confer a worse prognosis - the crescents are cellular and no interstitial inflammation was observed. In addition, hypercellularity, karyorrhexis and absence of chronic lesions reinforce the evidence of active renal involvement. As long as early and appropriate treatment is initiated, a favorable outcome might be anticipated.

\section{Would you perform a kidney biopsy in this patient?}

Kidney biopsy represents a useful diagnostic and prognostic tool in nephrology, providing valuable information regarding etiology, ongoing activity and potential reversibility of renal disease. However, serious complications may arise and potential risks should not outweigh expected benefits. No universal criteria are established, but most indications for this procedure are based on a composite of clinical and laboratorial findings, such as elevated serum creatinine, presence of increasing proteinuria and active urinary sediment - frail surrogates of kidney function and damage.

This premise is particularly relevant in patients with SLE and kidney involvement, since in most cases, clinical, serological or laboratory tests cannot accurately predict renal biopsy findings. ${ }^{4,11,12}$ Significant renal involvement (Class III, IV and V) has been documented in SLE patients with mild proteinuria, independently of abnormal urinary sediment ${ }^{13}$; on the other hand, active urinary sediment can be seen in flares with mild histological activity. Thus, the degree of proteinuria and the presence of hematuria are neither sensitive nor specific for renal disease activity or severity. ${ }^{14}$

Historically most guidelines recommended renal biopsy with proteinuria above $0.5 \mathrm{~g} /$ day with active urinary sediment ${ }^{12,15}$ or isolated proteinuria superior to $1 \mathrm{~g} /$ day. ${ }^{16}$ Although the threshold for 
performing a kidney biopsy is still not well defined in this population, most authors now agree that the presence of $>0.5 \mathrm{~g} /$ day of proteinuria, independent of the presence of microscopic hematuria and/or an increase in serum creatinine, is a reasonable indication, considering the potentially aggressive nature of this entity. ${ }^{1,4,5,16}$ Persistent increase in serum creatinine ( $>30 \%$ from baseline $)^{15,16}$, glomerular hematuria or leukocyturia (even if isolated) are also accepted as rational indications for kidney biopsy. ${ }^{11,16}$ Patients presenting with unexplained renal insufficiency with normal urinary findings can benefit as well from morphological evaluation, since it may differentiate active from chronic damage. 8,11

Given the absence of clinicopathological correlations in LN, keeping a low threshold for kidney biopsy in these patients seems reasonable. Therefore, biopsying this patient with $1 \mathrm{~g} /$ day of proteinuria was justified and proved to be enlightening. Renal morphology is essential not only to confirm (or exclude) the diagnosis of $L N$, but also to establish activity and chronicity of renal injury, guide therapeutic strategy, and provide prognostic information regarding potential response to treatment and of progression to end-stage renal disease (ESRD).

Disclosure of potential conflicts of interest: none declared

Authors' Contributions: equal authorship of Sara Barreto and Ana Domingos.

\section{References}

1. Wilhelmus S, Bajema IM, Bertsias GK et al. Lupus nephritis management guidelines compared. Nephrol Dial Transplant. 2016 Jun; 31(6): p. 904-13.

2. Rijnink EC, Teng YK, Kraaij T, Dekkers OM, Bruijn JA, Bajema IM. Validation of the Systemic Lupus International Collaborating Clinics classification criteria in a cohort of patients with full house glomerular deposits. Kidney Int. 2018 Jan; 93(1): p. 214-20.

3. Bajema IM, Wilhelmus S, Alpers CE et al. Revision of the International Society of Nephrology/ Renal Pathology Society classification for lupus nephritis: C larification of definitions, and modified National Institutes of Health activity and chronicity indices. Kidney Int. 2018 Apr; 93(4): p. 789-96.
4. Moroni G, Depetri F, Ponticelli C. Lupus nephritis: When and how often to biopsy and what does it mean? J Autoimmun. 2016 Nov; 74: p. 27-40.

5. Almaani S, Meara A, Rovin BH. Update on I upus n ephritis. Clin J Am Soc Nephrol. 2017 May 8; 12(5): p. 825-35.

6. Rijnink EC, Teng YK, Wilhelmus $S$ et al. Clinical and $\mathrm{h}$ istopathologic $\mathrm{c}$ haracteristics a ssociated with $r$ enal o utcomes in I upus $n$ ephritis. Clin J Am Soc Nephrol. 2017 May 8; 12(5): p. 734- 43.

7. Tesar V, Hruskova Z. Understanding $\mathrm{h}$ istolopathologic c haracteristics to $\mathrm{p}$ redict $r$ enal o utcomes in I upus $n$ ephritis. Clin J Am Soc Nephrol. 2017 May 8; 12(5): p. 711- 2.

8. Navarro D, Ferreira AC, Viana H, Carvalho F, Nolasco F. Índices morfológicos na nefrite I úpica: Orientação prognóstica? Um estudo e etrospetivo. Acta Med Port. 2019 Oct; 32(10).

9. Pagni F, Galimberti S, Galbiati E et al. Tubulointerstitial lesions in lupus nephritis: International multicentre study in a large cohort of patients with repeat biopsy. Nephrology (Carlton). 2016 Jan; 21(1): p. 35-45.

10. Almaani S, Prokopec SD, Zhang J et al. Rethinking I upus $\mathrm{n}$ ephritis $\mathrm{c}$ lassification on a m olecular Level. J Clin Med. 2019; 8(10): p. 1524.

11. Bertsias GK, Tektonidou M, Amoura Z et al. Joint European League Against Rheumatism and European Renal Association-European Dialysis and Transplant Association (EULAR/ERA-EDTA) recommendations for the management of adult and paediatric lupus nephritis. Ann Rheum Dis. 2012 Nov; 71(11): p. 1771-82.

12. Gordon C, Amissah-Arthur MB, Gayed M et al. The British Society for Rheumatology guideline for the management of systemic lupus erythematosus in adults. Rheumatology (Oxford). 2018 Jan 1; 57(1): p. e1-e45.

13. Christopher-Stine L, Siedner M, Lin J et al. Renal biopsy in lupus patients with low levels of proteinuria. J Rheumatol. 2007 Feb; 34(2): p. 332-5.

14. Ayoub I, Birmingham D, Rovin B, Hebert L. Commentary on the $c$ urrent $g$ uidelines for the $d$ iagnosis of I upus $n$ ephritis $f$ lare. Curr Rheumatol Rep. 2019 Feb 27; 21(4): p. 12.

15. Hahn BH, McMahon MA, Wilkinson A et al. American College of Rheumatology guidelines for screening, treatment, and management of lupus nephritis. Arthritis Care Res (Hoboken). 2012 Jun; 64(6): p. 797-808.

16. van Tellingen A, Voskuyl AE, Vervloet MG et al. Dutch guidelines for diagnosis and therapy of proliferative lupus nephritis. Neth J Med. 2012 May; 70(4): p. 199-207.

\section{Correspondence to:}

Mário Góis, MD

Laboratory of Renal Morphology, Hospital Curry Cabral - Centro Hospitalar e Universitário de Lisboa Central, Lisboa, Portugal

E-mail:mario.gois@chlc.min-saude.pt 